\title{
Cross-trial priming in visual search for singleton conjunction targets: Role of repeated target and distractor features
}

\author{
THOMAS GEYER \\ Ludwig-Maximilians-Universität, Munich, Germany \\ HERMANN J. MÜLLER \\ Ludwig-Maximilians-Universität, Munich, Germany \\ and Birkbeck College, University of London, England \\ and \\ JOSEPH KRUMMENACHER \\ Ludwig-Maximilians-Universität, Munich, Germany
}

\begin{abstract}
Kristjánsson, Wang, and Nakayama (2002) demonstrated that visual search for conjunctively defined targets can be substantially expedited ("primed") when target and distractor features are repeated on consecutive trials. Two experiments were conducted to examine whether the search response time (RT) facilitation on target-present trials results from repetition of target-defining features, distractor features, or both. The experiments used a multiple conjunctive search paradigm (adapted from Kristjánsson et al., 2002), in which the target and distractor features were varied (i.e., repeated) independently of each other across successive trials. The RT facilitation was numerically largest when both target and distractor features were repeated, but not significantly larger than that when only distractor features were repeated. This indicates that cross-trial priming effects in conjunctive visual search result mainly from the repetition of distractor, rather than target, features.
\end{abstract}

In the visual search paradigm, observers' task is to discern the presence of a target element among varying numbers of nontarget (distractor) elements. How efficiently a target can be detected has been found to depend on specific properties of the search display (see Wolfe, 1998, for a review) - in particular, whether the target differs from the distractors in a single feature or in a conjunction of different features, each of which is separately present in the distractors. However, even in the latter case, search can be surprisingly efficient. Since Treisman's proposal of her influential feature integration theory (FIT; Treisman \& Gelade, 1980), several mechanisms have been considered to account for how efficient detection of feature conjunction targets may be achieved. Although most accounts assume some form of top-down guidance of visual search (based on knowledge of the target-defining features), recent work has pointed to the potential role of bottom-up priming mechanisms (see the review below). The present experiments strengthen the case for bottom-up priming, and also show that priming is based mainly, though not

This research was supported by Deutsche Forschungsgemeinschaft Grant FOR 480/1-1. Correspondence concerning this article should be addressed to T. Geyer, Department Psychologie, Institut für Allgemeine und Experimentelle Psychologie, Ludwig-Maximilians-Universität, Martiusstrasse 4, 80802 Munich, Germany (e-mail: thomasgeyer@gmx.net). exclusively, on the repetition of distractor rather than target features.

\section{Efficient Visual (Conjunction) Search}

FIT (Treisman \& Gelade, 1980) characterizes target detection as a two-stage process. At the first, "preattentive" stage, the basic perceptual features of objects (e.g., color) are coded by dimension-specific modules of analyzers operating across the visual field. Spatiotopic feature maps are formed by each module, registering an object's feature value(s) within a particular dimension (e.g., red, green, etc., within the color module). At the second stage, spatial attention focuses on a position within a master map of locations (which signals where registered features occur within the field, but not their individual values) in order to retrieve and combine, within and across dimensions, the various features recorded at that position in the feature maps. This process leads to the creation of a temporary object representation referred to as an object file, which can be used to access stored knowledge for object recognition.

FIT proposes two distinct types of search behavior, depending on whether the target to be detected in a visual search display is defined by a unique feature in a given dimension (e.g., the only red element among blue elements) or a unique conjunction of features (e.g., the only red and vertical element among red horizontal and blue vertical elements). In a single-feature search, activity from a 
single feature map will signal the presence of the target, enabling it to be detected rapidly and independently of the number of display elements. This type of search behavior is referred to as parallel. In contrast, in feature conjunction search, serial focal-attentional inspection of element locations on the master map is necessary in order to bind the features together correctly, leading to search RTs that increase linearly with the number of elements. This type of search is referred to as serial.

However, following the original proposal of FIT, a number of findings were reported that challenged the strong assumption of a dichotomy between serial and parallel search processes. One finding was that of subset search (see, e.g., Bacon \& Egeth, 1997; Egeth, Virzi, \& Garbart, 1984; Friedman-Hill \& Wolfe, 1995; Kaptein, Theeuwes, \& van der Heijden, 1995) - that is, of significantly facilitated search for conjunctively defined targets (e.g., a red vertical target among red horizontal and blue vertical targets) when observers could effectively limit their search to a subset of the display elements (e.g., the red elements; within this subset, the target is the only vertical element, and thus is effectively defined by a unique orientation feature).

Many conjunction searches have been found to produce relatively shallow, or even flat, search latency slopes (which are typically associated with parallel search). These include searches for targets defined by shape and binocular disparity (Enns \& Rensink, 1991; He \& Nakayama, 1992; Ramachandran, 1988); by color and direction of motion; by binocular disparity and color, size, spatial frequency, and contrast (Nakayama \& Silverman, 1986); by shape and direction of motion (Driver, McLeod, \& Dienes, 1992; Kingstone \& Bischof, 1999; von Mühlenen \& Müller, 2001); and by color and orientation (Kaptein et al., 1995; Kristjánsson, Wang, \& Nakayama, 2002; Wang, Kristjánsson, \& Nakayama, 2001; Wolfe, Cave, \& Franzel, 1989).

To account for these discrepant findings (in relation to the original FIT), a revised FIT (Treisman \& Sato, 1990) assumes inhibitory connections between individual feature maps and the master map of locations. If the target and distractor (i.e., nontarget) features are known in advance, locations on the master map linked with distractor features can be actively inhibited, thereby enabling efficient search. Other accounts of visual search, such as guided search (Wolfe, 1994; Wolfe et al., 1989) and attentional engagement theory (Duncan \& Humphreys, 1989, 1992; see also Humphreys \& Müller, 1993), have proposed alternative mechanisms for efficient conjunction search, in particular top-down facilitation of target-defining features (rather than inhibition of distractor features), as well as the grouping and parallel rejection of distractors sharing features.

Furthermore, with singleton search tasks in which the features defining the target on a given trial are not known in advance, the mere repetition of the target features on consecutive trials can improve search efficiency (in terms of the base response time [RT] - i.e., the $y$-intercept of the function relating RT to display size; see, e.g., Maljkovic
\& Nakayama, 1994, 1996, 2000; McPeek, Maljkovic, \& Nakayama, 1999; Wang, Kristjánsson, \& Nakayama, 2005). In particular, in a singleton feature (i.e., pop-out) search task, Maljkovic and Nakayama $(1994,1996)$ found short-term priming for both the color and the position of the target: RTs were reduced when either the color or the position of the target was repeated, relative to conditions in which these target attributes were not repeated. Such RT advantages were evident for the last five to eight trials and were not found to be subject to top-down control (Maljkovic \& Nakayama, 1994, 2000; but see Müller, Krummenacher, \& Heller, 2004, and Müller, Reimann, \& Krummenacher, 2003, for conflicting evidence).

Recently, Kristjánsson, Wang, and Nakayama (2002; Wang et al., 2001) have also demonstrated priming in singleton conjunction search (see also Weidner, Pollmann, Müller, \& von Cramon, 2002). Kristjánsson et al. used a multiple conjunctive search paradigm, in which the target and distractors could change their features within sequences of trials. The participants' task was to detect a uniquely oriented red target, which was presented among differently oriented red and similarly oriented green distractors. Thus, on one trial, the orientation of the target could be horizontal when it appeared among vertical red and horizontal green distractors; on the next trial, it could be vertical in orientation and presented among horizontal red and vertical green distractors. The experiment comprised four major conditions: (1) In the conjunction condition, the target never changed its orientation - that is, it was always vertical; as a result, priming was expected to be large, leading to the fastest overall search RTs. (2) In the switch condition, the target changed its orientation predictably from horizontal to vertical, and vice versa, from one trial to the next; search performance was expected to be worse, because the orientation of the target was never repeated (i.e., there was no priming). (3) In the streak condition, the target also changed orientation between horizontal and vertical, but its orientation remained constant for longer streaks of trials; as a result, search performance was expected to be better than in the switch condition. (4) In the random condition, the target changed its orientation randomly between horizontal and vertical from one trial to the next, and again performance was expected to be superior to that in the switch condition. The results showed the expected pattern: Search RTs were fastest in the conjunction condition, intermediate in the streak and random conditions, and slowest in the switch condition (in terms of the $y$-intercepts of the functions relating RT to the number of elements in the display). However, when only RTs in the last few trials of a streak (Trials 6-8) were compared with the trial $n$ RT in the conjunction condition, search performance showed no quantitative difference between the two conditions. From this, the authors concluded that priming alone can account for the search RT benefits found in the conjunction condition - and, by extension, for efficient conjunctive search in general.

Interestingly, in the streak condition, priming was evident not only on target-present, but also on target-absent 
trials. This led Kristjánsson et al. (2002) to assume that (facilitatory) priming might result from faster perceptual grouping of distractor elements, which, in turn, might lead to faster decisions when the target was present or absent, because of faster discernment of target presence against the background of homogeneous, grouped distractors. However, for target-present trials, Kristjánsson et al. alternatively considered the possibility that facilitatory priming might be the result of repetition of the target features alone (see Maljkovic \& Nakayama, 1994, Experiment 8). Applied to their findings, repetition priming by the target might have annulled the effect of the repeated distractor orientation, so that distractor repetition would have had only a marginal effect (see Kristjánsson et al., p. 47).

There is, however, one shortcoming with this proposal. Because the orientations of both the target and the distractors were repeated in the streak condition, Kristjánsson et al. (2002) were unable to dissociate the effects of the repeated target and distractor orientations, and thus could not distinguish whether the mechanism of facilitatory priming was target or distractor based. The present experiment was designed to resolve this issue: Target and distractor features were varied independently of each other across consecutive trials, permitting the effects of repeated target and distractor features on priming in conjunctive visual search to be disentangled.

Given the evidence of facilitatory priming on targetabsent trials (Kristjánsson et al., 2002), it seems reasonable to assume that the same distractor-based priming mechanism is also at work on target-present trials; on the latter, however, an additional, target-based mechanism may come into play as well. (This theory is more parsimonious than the possibility considered by Kristjánsson et al., that the distractor-based effect is overridden by target-based priming on target-present trials.) Therefore, the present experiments sought evidence for how much the facilitatory priming on target-present trials is dependent on the repetition of distractor, rather than target, features.

\section{The Present Experiment}

The relative contributions of repeated distractor and target features to priming in conjunction search were assessed in two experiments, 1A and 1B. Both incorporated a multiple conjunctive search paradigm adapted from Kristjánsson et al. (2002): Observers were presented with displays of colored bar stimuli (see Figure 1 for an example) and had to discern the presence or absence of a singleton target defined by a conjunction of color (constant across trials) and bar orientation (variable across trials). More precisely, the red, uniquely oriented target could change its orientation from one trial to the next. The distractors were red and green in color. Red distractors had an orientation different from, and green distractors the same as, the target.

Experiments $1 \mathrm{~A}$ and $1 \mathrm{~B}$ differed in the number of possible target orientation alternatives, so we could examine the possible influence of target uncertainty on targetbased priming effects (for our rationale, see below). In Experiment 1A, the orientation of the (red) target was

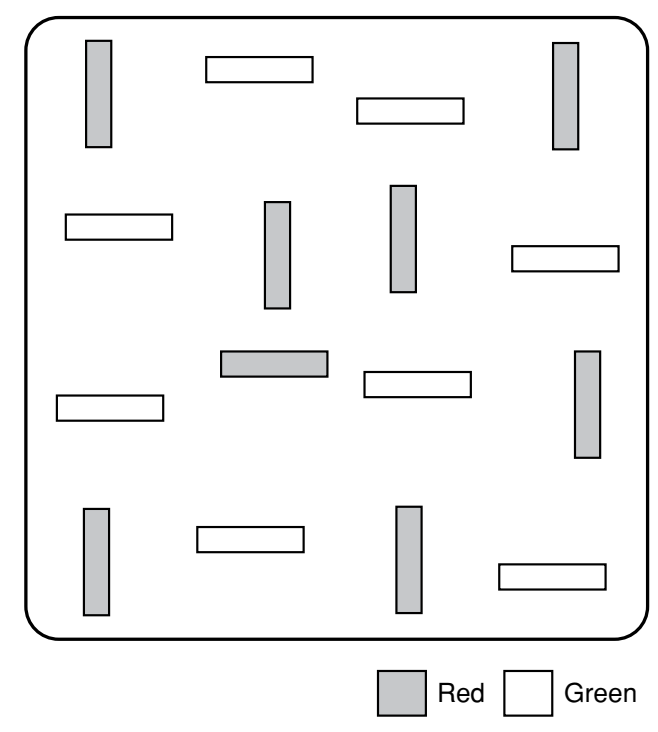

Figure 1. Example of a search display (with 16 elements) used in Experiments 1A and 1B. Participants' task was to detect a uniquely oriented red target bar, which was presented among differently oriented red distractors (relative to the target) and same-oriented green distractors. (In the figure, the target is the only red horizontal bar.)

either horizontal or vertical; that is, there were two target orientation alternatives. If the target was horizontal, the green distractors were horizontal and the red distractors were either vertical or oblique (tilted $45^{\circ}$ to either the left or the right). If the target was vertical, the green distractors were vertical and the red distractors were either horizontal or oblique. In Experiment 1B, the orientation of the target could be horizontal, vertical, or oblique (once again, tilted $45^{\circ}$ to the left or the right); that is, there were four target orientation alternatives. If the target was horizontal, the green distractors were horizontal and the red distractors were vertical; if the target was vertical, the green distractors were vertical and the red distractors horizontal. If the target was oblique, the green distractors were oblique in the same direction, and the red distractors could be either horizontal or vertical.

Given the variability of element orientations across trials, the following cross-trial transition conditions were realized in both experiments: repetition on consecutive trials of (1) both target and red distractor orientation, (2) target orientation alone, (3) red distractor orientation alone, and (4) neither target nor red distractor orientation. This design contrasts with that of Kristjánsson et al. (2002), who utilized only Conditions 1 and 4 . Thus, by varying target and (red) distractor repetition independently of each other, it became possible to determine the relative strengths of targetand distractor-based priming effects on target-present trials (and to compare these with the distractor-based priming effects on target-absent trials).

The logic of this determination was as follows. Priming was assessed by comparing target detection RTs in each of the three "repetition" conditions (i.e., repetition of target 
orientation only, distractor orientation only, or both) with the RTs in the baseline condition, in which neither the target nor the distractor orientation was repeated. It seemed reasonable to expect facilitation (relative to the baseline) to be at maximum when both target and distractor orientations were repeated (especially if target repetition makes a contribution over and above that of distractor repetition; in the most simple case, this would occur if both types of repetition had additive effects). This (expected) maximum priming effect could then be compared and contrasted with the effects when either target or distractor orientation was repeated alone.

If priming is due to repetition of the target orientation alone, the facilitatory effect of a repeated target should by itself be equivalent to the maximum priming effect. In contrast, if priming is determined by repetition of the distractor orientation alone, the facilitatory effect of a repeated distractor orientation should equal the maximum priming effect. However, if facilitatory priming is dependent on the repetition of both target and distractor orientations, a substantial RT advantage would be expected only when both target and distractor orientations are repeated.

Conceivably, target-based priming effects might depend on the number of alternative target orientations (target uncertainty), and distractor-based effects might depend on the number of alternative distractor orientations (distractor uncertainty). For example, the greater the number of target alternatives (i.e., the greater the amount of information gained by detecting a particular target orientation on a given trial), the greater the priming effect. To investigate the possible effects of target uncertainty, which modulates target-based priming effects, and distractor uncertainty, which modulates distractor-based priming effects, the numbers of alternative target and (red) distractor orientations were varied between Experiments 1A and 1B: In Experiment 1A, there were two alternatives for the target and four for red distractors; in Experiment 1B, there were four alternatives for the target and two for red distractors. Thus, if priming effects are modulated by uncertainty, one would expect target-based priming to be greater in Experiment 1B than in 1A and distractor-based priming to be greater in Experiment 1A than in 1B.

\section{METHOD}

\section{Participants}

Ten observers participated in Experiment 1A ( 7 females and 3 males, ranging from 21 to 43 years of age), and 10 new observers in Experiment 1B (4 females and 6 males, ranging from 22 to 39 years of age). All observers were naive about the intentions behind the study and reported normal or corrected-to-normal vision. They were paid $€ 8.00$ per session.

\section{Apparatus}

The experiments were conducted in a dimly lit laboratory to minimize reflections on the monitor. Stimulus presentation and RT measurement were controlled by a standard PC (a 75-MHz Pentium I). Stimuli were presented (at a frame rate of $60 \mathrm{~Hz}$ ) on a 17-in. color monitor with a resolution of $640 \times 480$ pixels. The observers viewed the monitor from a distance of approximately $60 \mathrm{~cm}$, maintained by the use of a chinrest. They responded "target present" and "target absent" by pressing the right and left buttons of a serial Microsoft mouse, with the track ball removed to improve timing accuracy (Segalowitz \& Graves, 1990).

\section{Stimuli}

The stimuli, which were modeled after those used by Kristjánsson et al. (2002), are depicted schematically in Figure 1. Each search display comprised either 4,8 , or 16 bar elements in various orientations, distributed randomly in the cells of an invisible $4 \times 4$ matrix. The matrix measured $14.9^{\circ} \times 14.9^{\circ}$ of visual angle, and the bars were $1.7^{\circ} \times 0.4^{\circ}$ in size, whether they were oriented horizontally, vertically, or obliquely ( $45^{\circ}$ left- or right-tilted). The arrangement of the bars within the display matrix was slightly jittered, with the horizontal and vertical distances between adjacent bars varying from $1.3^{\circ}$ to $2.7^{\circ}$. The bars were either red $\left(8.9 \mathrm{~cd} / \mathrm{m}^{2}\right)$ or green $\left(9.4 \mathrm{~cd} / \mathrm{m}^{2}\right)$. The background was essentially black and had a luminance of $0.5 \mathrm{~cd} / \mathrm{m}^{2}$. A white fixation cross was presented at the start of each trial in the display center; it was $0.5^{\circ} \times 0.5^{\circ}$ in size, with a luminance of $13.7 \mathrm{~cd} / \mathrm{m}^{2}$. Error feedback was given via a $1000-\mathrm{Hz}$ tone that sounded for $100 \mathrm{msec}$.

\section{Design and Procedure}

Figure 2 presents all possible target and distractor orientations used Experiments 1A (upper panel) and 1B (lower panel). The target, if present, was always a red bar and could change its orientation across trials. The distractors were red and green bars and changed their orientations in accordance with the target.

In Experiment 1A, a horizontal target appeared among horizontal green and either vertical (10.0\% of all trials), left-tilted (10.0\%), or right-tilted $(10.0 \%)$ red distractors, and a vertical target appeared among vertical green and either horizontal $(10.0 \%)$, left-tilted $(10.0 \%)$, or right-tilted $(10.0 \%)$ red distractors. In the absence of a target, the six possible distractor combinations were horizontal red and vertical green distractors $(6.6 \%)$; vertical red and horizontal green distractors $(6.6 \%)$; or left- or right-tilted red distractors, appearing with either horizontal or vertical green distractors (four combinations, $6.6 \%$ each).

In Experiment 1B, a horizontal target was presented among horizontal green and vertical red distractors $(10.0 \%$ of all trials). If the target was vertical, it appeared among vertical green and horizontal red distractors $(10.0 \%)$. For this experiment, the target could also be left- or right-tilted, in which case it was presented among green distractors with the same oblique orientation as the target and either horizontal or vertical red distractors (four combinations, $10.0 \%$ each). On target-absent trials, horizontal red distractors were presented with either vertical, left-tilted, or right-tilted green distractors $(6.6 \%$ each), or vertical red distractors were presented with either horizontal, left-tilted, or right-tilted green distractors $(6.6 \%$ each).

Experiments 1A and 1B consisted of 2,160 experimental trials each - that is, 360 trials for each display size $(4,8$, or 16 elements) $\times$ target (present or absent) combination. Each display size condition consisted of 360 mixed pairs of trials, representing six replications of all 36 possible cross-trial contingencies on (consecutive) target-present trials (e.g., horizontal target among horizontal green and vertical red distractors, followed by horizontal target among horizontal green and right-tilted red distractors), and four replications of all 36 cross-trial contingencies on target-absent trials (e.g., horizontal green and vertical red distractors followed by vertical green and horizontal red distractors). Across the target-present/ -absent trials, each cross-trial contingency was realized 10 times. Note that the ratio of target-present to target-absent trials was 60:40, in order to take account of the greater number of major cross-trial transition conditions on target-present than on target-absent trials (4 vs. 2; see below).

Since the last trial of a pair formed the first trial of the next pair, after randomization, each cross-trial contingency should be represented 20 (rather than just 10) times in each display size condition. However, for the same reason, a target-present trial could also follow a target-absent 

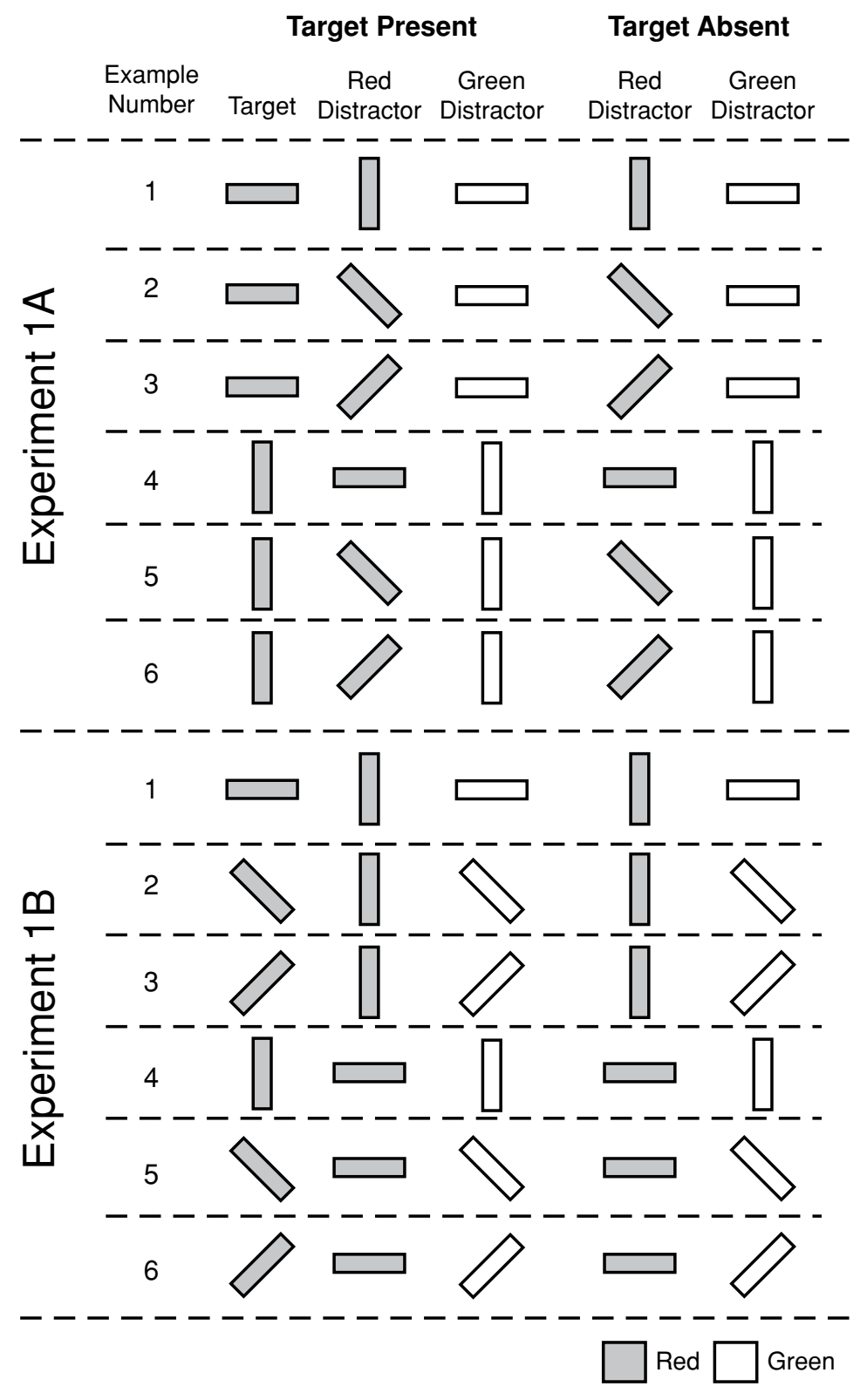

Figure 2. Illustration of all possible combinations of target and distractor orientations on target-present and -absent trials in Experiments 1A (upper panel) and 1B (lower panel).

trial, and vice versa. Because the aim of the study was to analyze the effects of repeated target and distractor orientations, which required pairs of target-present and pairs of target-absent trials, mixed-trial pairs (target-present following target-absent trials, or vice versa) were discarded from further analysis (about $25 \%$ of all trials). Of the remaining trials, $60 \%$ were target-present and $40 \%$ target-absent. Thus, each of the 36 different cross-trial contingencies on target-present trials was repeated at least six times, and each of the 36 contingencies on target-absent trials was repeated at least four times.

The 36 different pairs of target-present trials could be classed in terms of four major cross-trial transition conditions: $s T s D$, for same-oriented target, same-oriented red distractors (6/36 cross-trial contingencies in both experiments); $s T d D$, for same-oriented target, differently oriented red distractors (Experiment 1A, 12/36; Experi- ment 1B, 4/36); $d T s D$, for differently oriented target, same-oriented red distractors (Experiment 1A, 4/36; Experiment 1B, 12/36); and $d T d D$, for differently oriented target, differently oriented red distractors $(14 / 36)$. The 36 pairs of target-absent trials could be classed in terms of two major conditions: $s D$, same-oriented red distractors (Experiment 1A, 10/36; Experiment 1B, 18/36), and $d D$, differently oriented red distractors (Experiment 1A, 26/36; Experiment 1B, 18/36). See also Table 1, which summarizes separately for Experiments $1 \mathrm{~A}$ and $1 \mathrm{~B}$ both the probabilities and observation numbers for the transitions on successive target-present trials (sTsD, sTdD, $\mathrm{dTsD}$, and $\mathrm{dTdD}$ ) and successive target-absent trials (sD and $\mathrm{dD}$ ).

At the beginning of a trial, a fixation cross was presented in the center of the monitor for $800 \mathrm{msec}$, followed by a blank interval of $200 \mathrm{msec}$. Thereafter, the search stimuli appeared and remained vis- 
Table 1

Probabilities of Target and Distractor Orientation Repetitions/Changes on Consecutive Trials (Cross-Trial Transitions), Presented Separately for Target-Present and -Absent Trials in Experiments $1 \mathrm{~A}$ and $1 \mathrm{~B}$

\begin{tabular}{cccccc}
\hline & \multicolumn{2}{c}{ Experiment 1A } & & \multicolumn{2}{c}{ Experiment 1B } \\
\cline { 2 - 3 } \cline { 5 - 6 } Transition & Probability & Observations & & Probability & Observations \\
\hline Target present & & & & & \\
sTsD & $6 / 36$ & 36 & & $6 / 36$ & 24 \\
sTdD & $12 / 36$ & 72 & & $4 / 36$ & 72 \\
dTsD & $4 / 36$ & 24 & & $12 / 36$ & 84 \\
dTdD & $14 / 36$ & 84 & & $14 / 36$ & 72 \\
Target absent & & & & $18 / 36$ \\
sD & $10 / 36$ & 40 & & $18 / 36$ & 72 \\
dD & $26 / 36$ & 104 & & 104 \\
\hline
\end{tabular}

Note-sTsD, same-oriented target and same-oriented red distractors; sTdD, sameoriented target and differently oriented distractors; dTsD, differently oriented target and same-oriented distractors; dTdD, differently oriented target and differently oriented distractors; $\mathrm{sD}$, same-oriented distractors; $\mathrm{dD}$, differently oriented distractors. Each type of intertrial transition was presented six and four times on target-present and target-absent trials, respectively; for example, in Experiment 1A, this resulted in 36 and 40 observations for the STsD and sD transitions, respectively.

ible until participants responded "target present" or "target absent" by pressing the right or left button of the computer mouse with the index finger of their right or left hand, respectively. When an observer had made an incorrect response (target miss or false alarm), he or she was alerted to the error by a brief computer-generated beep. The intertrial interval was $1,000 \mathrm{msec}$ after correct-response trials and 2,000 msec after an error signal. Within each experiment, all different cross-trial contingencies on target-present and -absent trials were presented in randomized order. The number of display elements remained the same within a block, but it varied randomly across blocks.

Experiments $1 \mathrm{~A}$ and $1 \mathrm{~B}$ were both run in two sessions, separated by a break of at least $1 \mathrm{~h}$. Each session consisted of 12 blocks of 5 (unrecorded) practice trials and 85 experimental trials. Before the beginning of the experiment, the observers performed a practice session of 75 trials (data not recorded).

In both Experiments 1A and 1B, the observers were instructed to respond as fast and as accurately as possible to the presence or absence of the uniquely oriented red bar.

\section{RESULTS}

For both experiments, the data from the practice block and from the five warm-up trials at the start of each experimental block were excluded from the analysis. For each experimental condition (display size $\times$ target), RTs outside the range of \pm 2.5 standard deviations $(S D)$ from the mean were discarded as outliers (overall, $2.8 \%$ of trials in Experiment 1A, 2.6\% in Experiment 1B). RTs for error trials were also excluded from the analysis $(2.8 \%$ and $2.9 \%$ of all trials in Experiments $1 \mathrm{~A}$ and $1 \mathrm{~B}$, respectively; for further details, see the accuracy results below). In examinations of the effects of repetition, the current trial may have been influenced by the preceding trial and also influenced the subsequent one. Therefore, responses on trials that preceded or followed an erroneous response were also not analyzed. In other words, repetition effects were analyzed only for two consecutive trials on which the responses were correct.

The results are presented in the following sections, first for overall RT and accuracy performance, then for the theoretically important effects of cross-trial transition in both target-absent (sD and dD) and -present (sTsD, sTdD, $\mathrm{dTsD}$, and $\mathrm{dTdD}$ ) trials, respectively.

\section{Overall RT Performance}

Figure 3 presents the group mean correct RTs, along with the error rates, in Experiments 1A (left-hand panel) and $1 \mathrm{~B}$ (right-hand panel) as a function of display size, presented separately for target-present and -absent trials. RTs were examined through a mixed-design ANOVA with three factors: experiment (1A or 1B; between subjects), display size $(4,8$, or 16 elements), and target (present or absent). This analysis revealed the main effects of display size $\left[F(2,18)=47.82, M S_{\mathrm{e}}=9,927, p<.01\right]$ and target $\left[F(1,9)=13.05, M S_{\mathrm{e}}=6,127, p<.01\right]$ to be significant, and all other effects to be nonsignificant. RTs increased with increasing display size (main effect of display size: 622,710 , and $838 \mathrm{msec}$ for the 4-, 8-, and 16-element displays, respectively), and target-present were faster than target-absent RTs (697 vs. $749 \mathrm{msec}$ ). Interestingly, there were no effects of experiment $\left[F(1,9)=0.34, M S_{\mathrm{e}}=\right.$ $116,586, p=.57]$, indicating that similar search processes operated in both Experiments 1A and 1B.

\section{Overall Response Accuracy}

Participants' individual error rates ranged between 1.0\% and $4.4 \%$ in Experiment $1 \mathrm{~A}$ and between $1.6 \%$ and $4.1 \%$ in Experiment 1B. The individual error rates were also analyzed in a mixed-design ANOVA, with experiment (1A or 1B; between subjects), display size (4, 8, or 16 elements), and target (present or absent) as factors. This ANOVA revealed the main effect of display size $[F(2,18)=11.13$, $\left.M S_{\mathrm{e}}=1.46, p<.01\right]$ and the two-way interaction between display size and target $\left[F(2,18)=20.78, M S_{\mathrm{e}}=2.82, p<\right.$ $.01]$ to be significant. More errors were made when display size increased (main effect of display size: $2.66 \%, 2.74 \%$, and $3.80 \%$ for the $4-, 8$-, and 16-element displays, respectively). However, this effect was due to a moderate increase in error responses on target-present trials (miss rates of 


\section{Experiment 1A}

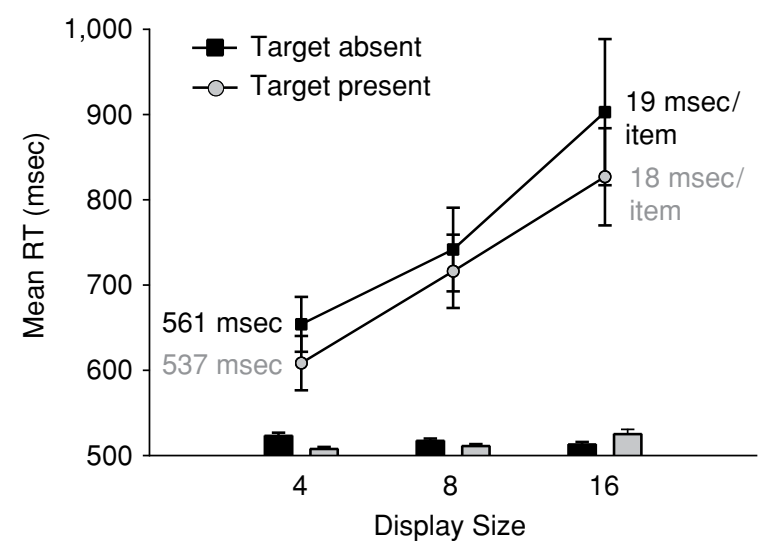

Experiment 1B

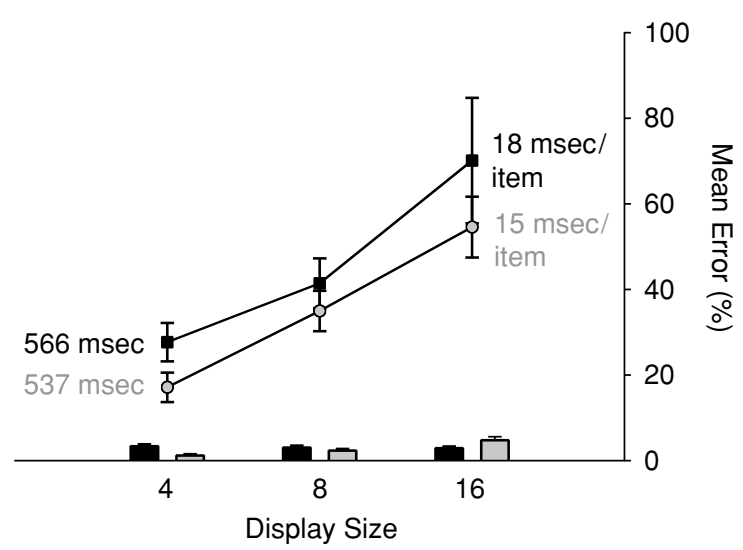

Figure 3. Group mean correct RTs with associated standard errors (compare lines with left $y$-axis) and group mean percentage errors (compare bars with right $y$-axis) for target-present and -absent trials as a function of display size, presented separately for Experiments 1A (left panel) and 1B (right panel). The number to the left of each line represents the $y$-intercept (in milliseconds), the number to the right the search rate (in milliseconds per element) of a particular RT/display size function.

$1.35 \%, 2.26 \%$, and $4.88 \%$ for the $4-, 8$-, and 16 -element displays, respectively) relative to target-absent trials (false alarm rates of $3.97 \%, 3.22 \%$, and $2.73 \%$, respectively), which accounts for the display size $\times$ target interaction. Thus, there was little overall indication that the main effects of display size and target (and the nonsignificant effect of experiment) found for RTs were confounded by speedaccuracy trade-offs.

\section{Cross-Trial Analysis of Target-Absent RTs}

Figure 4 presents, for Experiments $1 \mathrm{~A}$ and $1 \mathrm{~B}$, the group mean correct RTs on target-absent trials as a function of display size, separately for same (sD) and different (dD) red distractor orientation cross-trial transitions. Furthermore, for each display size condition, Figure 4 gives the difference in RTs of the $\mathrm{sD}$ versus the $\mathrm{dD}$ (baseline) condition.

To examine the effect of repeated distractor orientation, the target-absent RTs were analyzed in a separate mixed-design ANOVA with the factors experiment (1A or $1 \mathrm{~B}$; between subjects), display size $(4,8$, or 16$)$, and transition ( $\mathrm{sD}$ or $\mathrm{dD})$. The main effect of display size was significant $\left[F(2,18)=27.29, M S_{\mathrm{e}}=8,944, p<.01\right]$ and due to a slowing of RTs with increasing display size (633, 707, and $850 \mathrm{msec}$ for 4-, 8-, and 16-element displays, respectively). Furthermore, there was a reliable main effect of transition $\left[F(1,9)=24.95, M S_{\mathrm{e}}=890, p<.01\right]$ : RTs were significantly reduced when the orientation of the red distractors was repeated rather than changed $(711 \mathrm{msec}$

\section{Experiment 1A (Absent)}

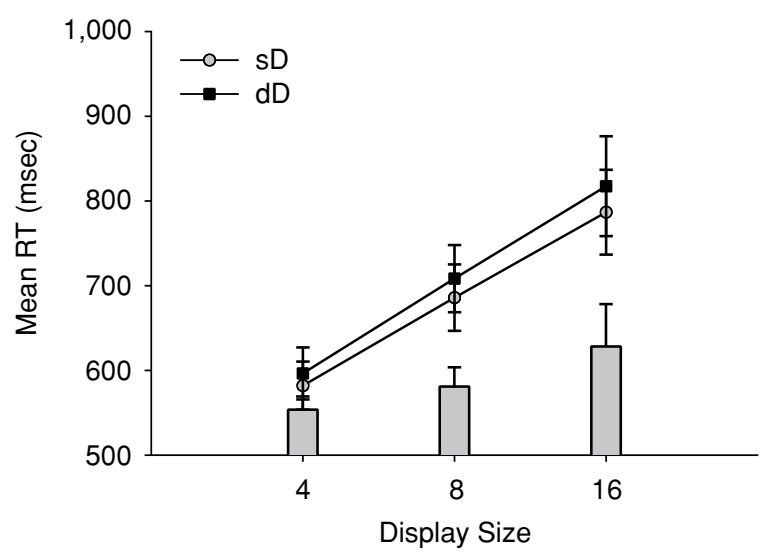

\section{Experiment 1B (Absent)}

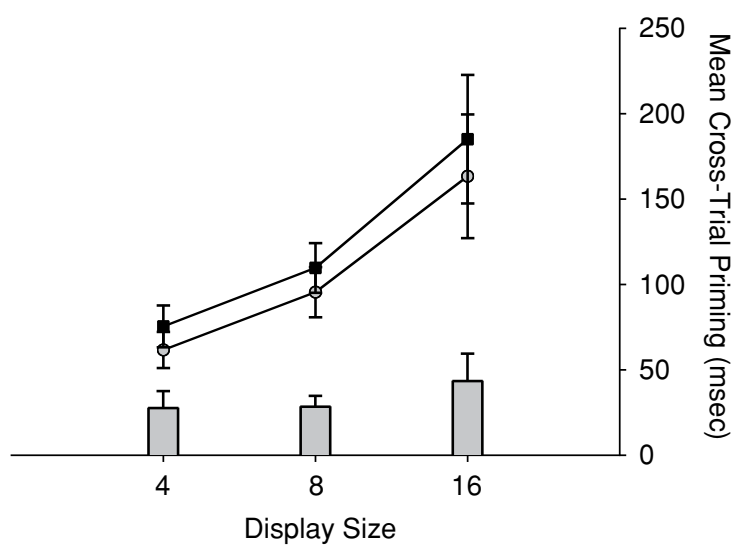

Figure 4. Mean correct target-absent RTs with associated standard errors (compare lines with left $y$-axis) and mean RT differences between the conditions (compare bars with right $y$-axis), presented as a function of display size separately for Experiments 1A (left) and 1B (right). In the line graphs, gray circles represent the same-oriented (sD) and black squares the differently oriented (dD) red distractor conditions. Cross-trial priming (gray bars) was assessed by comparing RTs in the $\mathrm{dD}$ (baseline) and sD conditions. 
[sD] vs. $750 \mathrm{msec}[\mathrm{dD}])$. No other effects were significant. Note, however, that there was a nonsignificant tendency for the RT facilitation due to distractor repetition to increase as a function of display size $[28,35$, and $54 \mathrm{msec}$ for 4-, 8-, and 16-element displays, respectively; transition $\times$ display size interaction: $F(2,18)=2.12, M S_{\mathrm{e}}=$ $440, .10<p<.15$ ].

\section{Cross-Trial Analysis of Target-Present RTs}

Figure 5 presents, for Experiments $1 \mathrm{~A}$ and $1 \mathrm{~B}$, the group mean correct RTs on target-present trials as a function of display size, presented separately by cross-trial transition: repetition of both target and (red) distractor orientations (sTsD), of target orientation alone (sTdD), of distractor orientation alone (dTsD), and of neither target nor distractor orientation (dTdD). Furthermore, for each display size condition, the differences in RT are given for the sTsD, sTdD, and dTsD conditions relative to the $\mathrm{dTdD}$ (baseline) condition. A mixed-design ANOVA with the factors experiment (1A or 1B; between subjects), display size $(4,8$, or 16 elements), and transition (sTsD, sTdD, $\mathrm{dTsD}$, or $\mathrm{dTdD}$ ) revealed significant main effects of display size $\left[F(2,18)=108.44, M S_{\mathrm{e}}=3,757, p<.01\right]$ and transition $\left[F(3,27)=12.50, M S_{\mathrm{e}}=676, p<.01\right]$, as well as a display size $\times$ transition interaction $[F(6,54)=2.89$, $\left.M S_{\mathrm{e}}=392, p<.02\right]$.

Search RTs increased as a function of the number of display elements (main effect of display size: 588, 684, and $789 \mathrm{msec}$ for 4-, 8-, and 16-element displays, respectively). Furthermore, RTs were fastest in the sTsD $(673 \mathrm{msec})$ and $\mathrm{dTsD}$ conditions $(674 \mathrm{msec})$, intermediate in the sTdD condition (692 msec), and slowest in the dTdD condition (708 msec) (main effect of transition). However, this effect of transition was dependent on the display size (display size $\times$ transition interaction): With 16-element displays, search RTs were fastest in the sTsD $(768 \mathrm{msec})$ and dTsD conditions ( $770 \mathrm{msec})$, intermediate in the sTdD condition (793 $\mathrm{msec})$, and slowest in the dTdD condition $(828 \mathrm{msec})$ (Tukey LSD tests revealed the fastest RTs to be significantly faster than the intermediate RTs, which in turn were significantly faster than the slowest RTs); thus, the ordering was the same as for the transition main effect. However, with 8 -element displays, observers responded fastest in the sTsD (671 msec) and dTsD (671 msec) conditions, and slowest in the sTdD (693 msec) and dTdD (701 msec) conditions. With 4-element displays, RTs were comparable in all transition conditions $(581,592,582$, and $597 \mathrm{msec}$ in the sTsD, sTdD, dTsD, and dTdD conditions, respectively); nevertheless, even here the pattern of RTs tended to be similar to those observed for the 8- and 16-element displays. Overall, the RT facilitation resulting from distractor, and to a lesser extent target, repetition increased as a function of display size: For the distractor repetition (dTsD) condition, facilitation was 16, 30, and $58 \mathrm{msec}$ in the 4-, 8-, and 16-element displays, respectively; for the target repetition (sTdD) condition, the respective values were 6,8 , and $35 \mathrm{msec}$. The pattern was also evident (though nonsignificant) in the target-absent trials.

\section{Comparison of Distractor Repetition Effects Between Target-Absent and Target-Present Trials}

To compare the facilitatory effects of distractor repetition between target-absent and -present trials, a further mixed-design ANOVA with the factors experiment (1A or $1 \mathrm{~B}$, between subjects), target (absent or present), display size $(4,8$, or 16 elements), and transition ( $\mathrm{sD}$ or $\mathrm{dD}$; i.e., for

\section{Experiment 1A (Present)}

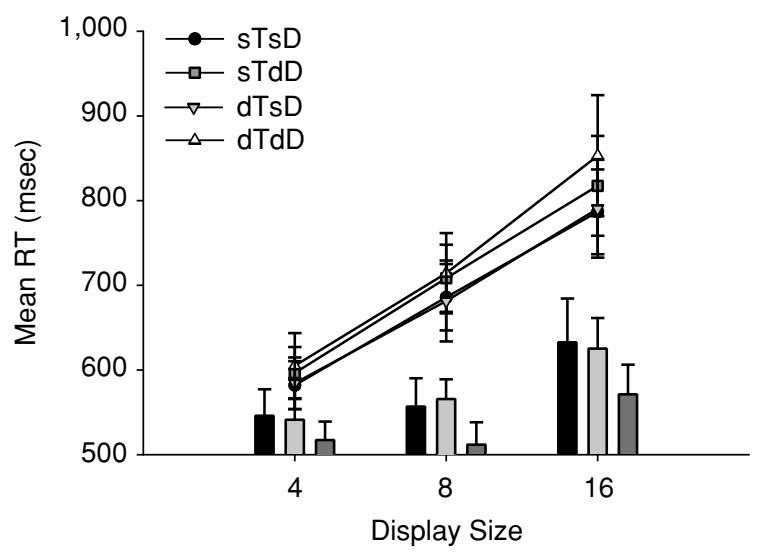

\section{Experiment 1B (Present)}

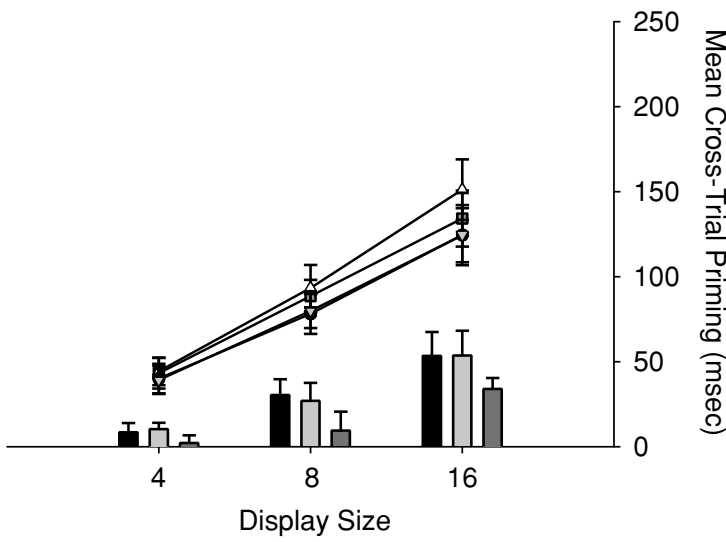

Figure 5. Mean correct target-present RTs with associated standard errors (compare lines with left $y$-axis), and mean RT differences between the conditions (compare bars with right $y$-axis), presented as a function of display size separately for Experiments 1A (left) and 1B (right). In the line graphs, black circles represent same-oriented targets and sameoriented red distractors across trials (sTsD), dark gray squares same-oriented targets and differently oriented distractors (sTdD), light gray triangles differently oriented target and same-oriented distractors (dTsD), and white triangles differently oriented targets and differently oriented distractors (dTdD). In the bar chart, cross-trial priming was assessed by comparing RTs in the dTdD (baseline) condition against those in, respectively, the sTsD (black bars), dTsD (light gray bars), and sTdD (dark gray bars) conditions. 
target-present trials, dTsD vs. dTdD) was carried out. This ANOVA revealed only the main effects of target $[F(1,9)=$ 8.30, $M S_{\mathrm{e}}=11,152, p<.02$; faster target-present than -absent RTs], display size $\left[F(2,18)=56.41, M S_{\mathrm{e}}=16,349\right.$, $p<.01$; RT increasing as a function of display size], and transition $\left[F(1,9)=33.41, M S_{\mathrm{e}}=2,389, p<.01\right.$; faster RTs for repeated than for nonrepeated distractor orientations] to be significant. Furthermore, the display size $\times$ transition interaction $\left[F(2,18)=7.88, M S_{\mathrm{e}}=796, p<\right.$ $.01]$ reached significance, indicating increased RT facilitation associated with distractor repetition as the display size increased. Importantly, none of the interactions involving target and transition were significant [target $\times$ transition interaction: $\left.F(1,9)=0.59, M S_{\mathrm{e}}=404, p>.45\right]$. That is, the RT facilitation deriving from distractor repetition was not significantly different between target-absent and -present trials (39 vs. $35 \mathrm{msec}$, respectively). (The small numerical difference is unsurprising, for two reasons: First, the target-present RTs were generally faster, by $40 \mathrm{msec}$, leaving somewhat less room for priming to become fully effective. In addition, for target-absent trials, the red target was replaced by an additional red distractor, giving rise to a stronger distractor grouping.)

\section{Effects of Target and Distractor Uncertainty}

None of the ANOVAs above revealed an effect of experiment; that is, no effect emerged of the variation in target versus distractor uncertainty across the two experiments (two target vs. four distractor alternatives in 1A, four target vs. two distractor alternatives in 1B). There were, however, some tendencies in the data that are worth mentioning. Doubling the number of target alternatives had no discernible effect on the RT facilitation deriving from target repetition (i.e., the RT difference between the dTdD and sTdD conditions), with overall facilitation of 17 and $16 \mathrm{msec}$ in Experiments $1 \mathrm{~A}$ and $1 \mathrm{~B}$, respectively. In contrast, doubling the number of distractor alternatives did tend to increase the RT facilitation deriving from distractor repetition (i.e., the RT difference between the $\mathrm{dD}$ and $\mathrm{sD}$ [target-absent trials] and dTdD and dTsD [target-present trials] conditions): On target-absent trials, the overall facilitation was 44 versus $33 \mathrm{msec}$ in Experiments $1 \mathrm{~A}$ and 1B, respectively, and on target-present trials it was 39 versus $30 \mathrm{msec}$ on target-present trials. [However, comparing distractor repetition effects between target-absent and -present trials, the ANOVA failed to reveal a significant experiment $\times$ transition interaction: $F(1,9)=0.74, M S_{\mathrm{e}}=1,834, p>.40$.]

Since priming effects can also accumulate across sequences of trials (see the introduction), the analysis of the trial $n$ RTs as a function of distractor orientation in the immediately preceding trial $n-1$ may have underestimated the comparative effects of repeated distractor orientation under the two uncertainty conditions (i.e., Experiments $1 \mathrm{~A}$ and $1 \mathrm{~B}$ ), because distractor priming may not have been fully effective after only a single repetition. If this is correct, then the fact that there was no overall RT difference between Experiments 1A and 1B (i.e., no significant effect of experiment in the ANOVAs above) could be due to two opposing effects associated with distractor uncertaintygreater magnitude of cross-trial priming, but reduced probability of repeated-distractor (i.e., priming) trials with high (Experiment 1A) relative to low (Experiment 1B) distractor uncertainty. In other words, although the priming effect may have been larger with high distractor uncertainty, the relevant trials (with repetition of a particular distractor orientation) were less likely to occur in Experiment 1A, leading to net priming effects equivalent to those with low distractor uncertainty in Experiment 1B, and consequently to equivalent overall RTs.

To test this theory more directly, we reanalyzed the trial $n$ RTs as a function of the (red) distractor orientation on trial $n-2$, with the trial $n-1$ distractor orientation identical to that on trial $n-2$, in an ANOVA with the factors experiment (1A or 1B; between subjects), target (absent or present), display size $(4,8$, or 16 elements), and transition ( $\mathrm{sD}$ or $\mathrm{dD}$; i.e., for target-present trials, $\mathrm{dTsD}$ vs. dTdD). (This analysis was limited to the influence of trial $n-2$, because the numbers of observations for preceding trials $n-3, n-4$, etc., were too small to permit statistical analyses.) This ANOVA did reveal a significant experiment $\times$ transition interaction $\left[F(1,9)=5.10, M S_{\mathrm{e}}=6,029\right.$, $p<.05]$ : As is illustrated in Figure 6, when the trial $n$ distractor orientation was repeated across a sequence of three trials, the facilitatory effect was larger in Experiment $1 \mathrm{~A}$ than in Experiment 1B, for both target-present (104 vs. $63 \mathrm{msec}$ ) and target-absent (105 vs. $58 \mathrm{msec}$ ) trials. This result confirms that greater cumulative cross-trial priming does occur with higher distractor uncertainty.

\section{DISCUSSION}

The present experiments were designed to examine the mechanisms of facilitatory priming in conjunctive visual search. The participants' task was to detect a uniquely oriented red target item (presented in $60 \%$ of all trials) among differently oriented red and same-oriented green distractor items. Unlike standard conjunctive search, in which the target and distractor features remain constant throughout the task (feature certainty), the present experiments used a multiple color-orientation conjunction search paradigm, in which both the target and the distractors could independently change their orientation features across consecutive trials. Thus, the number of orientation alternatives for the target could be different from the number of alternatives for the red distractors (in Experiment 1A, two vs. four alternatives, respectively, so distractor uncertainty $>$ target uncertainty; in Experiment 1B, four vs. two alternatives, so target uncertainty $>$ distractor uncertainty). With this manipulation, it was possible to decide (1) whether, and to what extent, facilitatory priming depends on the repetition of both target and distractor orientation, target orientation alone, or distractor orientation alone; and (2) to what extent priming is modulated by the degrees of target and distractor uncertainty.

The analysis of target-absent trials revealed that the repetition of the red distractors' orientation (sD) led to 


\section{Experiment 1A (Absent)}

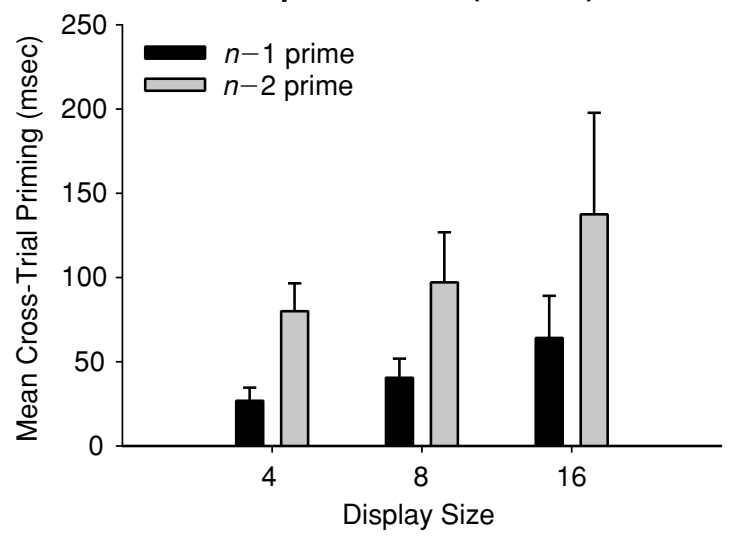

Experiment 1A (Present)

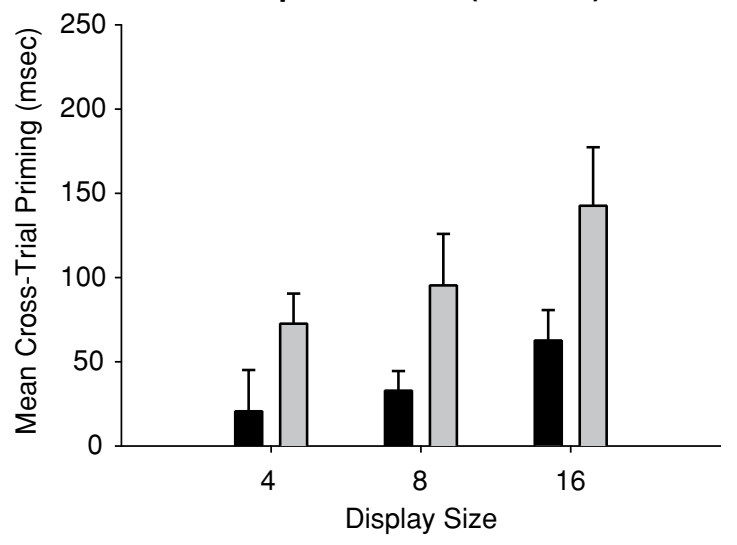

Experiment 1B (Absent)

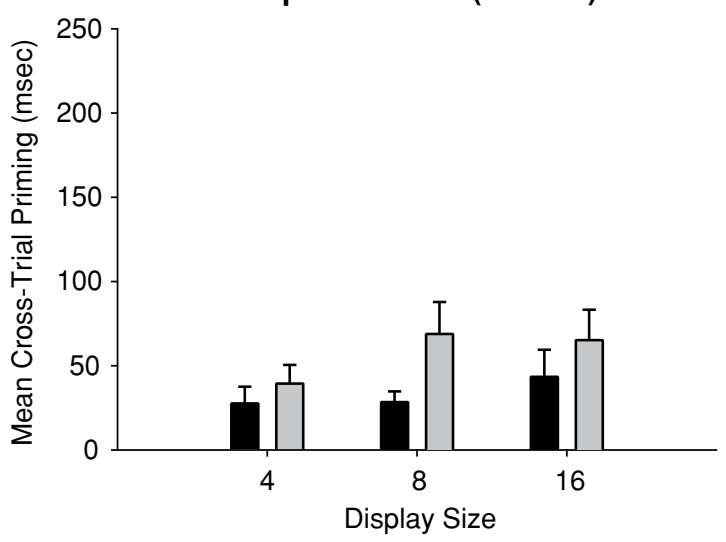

Experiment 1B (Present)

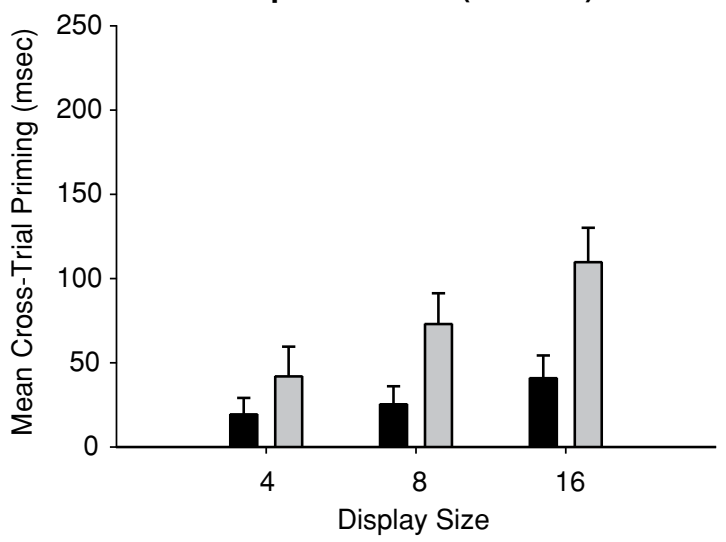

Figure 6. (Upper panels) For target-absent trials, mean RT differences and associated standard errors between the differently oriented (dD) and same-oriented (sD) red distractor conditions. (Lower panels) For target-present trials, mean RT differences and associated standard errors between the differently oriented target, differently oriented distractor (dTdD) and differently oriented target, same-oriented distractor (dTsD) conditions. All graphs are presented as a function of display size, separately for Experiments $1 \mathrm{~A}$ (left panels) and $1 \mathrm{~B}$ (right panels). Black bars represent the cross-trial priming when the trial $n$ red distractor orientation was the same as that on trial $n-1$ ( $n-1$ prime), and gray bars represent the priming when the trial $n$ red distractor orientation was the same as that on both trials $n-1$ and $n-2(n-2$ prime).

RT advantages of some $40 \mathrm{msec}$, across all display sizes, relative to a change of distractor orientation $(\mathrm{dD})$. This effect confirms that facilitatory priming can occur even in the absence of a target (see Kristjánsson et al., 2002). On target-present trials, the repetition of both target and distractor orientation ( $\mathrm{sTsD}$ ) expedited responses by $35 \mathrm{msec}$ on average, relative to the nonrepetition of target and distractor orientation (dTdD). Interestingly, the RT facilitation was not reduced from this level when only the distractor, not the target, orientation was repeated (dTsD). In contrast, the facilitatory effect resulting from repetition of the target but not the distractor orientation was comparatively small in magnitude $(16 \mathrm{msec})$. This pattern of results supports the view that priming in conjunctive visual search results mainly from the repetition of distractor rather than target orientation, a result that is in line with previous studies (see, e.g., Karni \& Sagi, 1993).

When distractor orientation was repeated, additional repetition of the target orientation had almost no extra effect; however, when only the target orientation was repeated, there was an effect, but it was less than half the size of the distractor orientation repetition effect. These results suggest a nonadditive, interactive account of distractor- and target-based priming effects, such that target repetition can only marginally enhance the (almost "saturated") effect of distractor repetition. The dominance of distractorbased priming may arise because distractor repetition allows faster perceptual grouping, permitting target presence or absence to be discerned more rapidly (among homogeneous, grouped distractors). Significantly, the present results do not rule out that some priming effect does result from the repetition of target orientation, consistent with the priming mechanisms discussed by Kristjánsson et al. (2002). However, the results demonstrate that the net priming effect is largely dependent on the repetition of distractor features (here, orientation). This finding is at variance with the possibility considered by Kristjánsson et al., that the priming effect of repeated target orientation may dominate (i.e., annul) the facilitatory effect of distractor repetition. 
Note, though, that the assumption of "grouping" (Kristjánsson et al., 2002) is not necessary to account for distractor-based priming, for alternative accounts based on guided search are also feasible. One such account is that considered by Hillstrom (2000) for target-based priming: Repetition of target-defining attributes (e.g., orientation) enhances target feature coding, which, in turn, expedites target detection. It may be possible to differentiate between these alternative explanations of distractor priming by considering where in visual processing the priming may arise. Hillstrom regarded the facilitation of target feature coding as a top-down effect, whereas Kristjánsson et al. considered the enhanced grouping of search distractors to occur at an intermediate, bottom-up-driven stage of processing. If priming is indeed based on top-down mechanisms, it is likely that the RT advantage resulting from repeated distractor orientation derives from the repetition of particular subsets (e.g., red) rather than of all (e.g., both red and green) of the distractors. In particular, since the target was defined as a uniquely oriented red element, participants may have top-down restricted their search to the samecolored distractors in order to look for an odd-one-out orientation element (i.e., a red item, but differently oriented relative to the rest). In this case, only the orientation of the red rather than the green distractors would matter. In contrast, if priming reflects bottom-up (grouping) mechanisms, RT facilitation should be evident only, or at least more strongly, when all (i.e., both red and green) distractor sets are repeated.

To decide between these two accounts, a control experiment was conducted that was designed to test the subset search hypothesis. ${ }^{1}$ In contrast to Experiments $1 \mathrm{~A}$ and 1B, the orientation of the red distractors was (orthogonally) coupled with that of the red target, and the orientation of the green distractors was varied independently of the target orientation. This control experiment revealed a significant RT advantage only when the red, not the green, distractors were repeated. This finding indicates that observers were able to limit their search to a particular subset of distractors. By implication, it suggests that the priming effects in Experiments 1A and 1B resulted from top-down enhanced processing (subset search) of relevant distractors (as in Hillstrom, 2000) rather than bottom-up expedited grouping (Kristjánsson et al., 2002). Top-down enhanced distractor processing would permit the presence of a differently oriented red element among red elements with a repeated orientation to be discerned more efficiently, possibly because the repeated distractor orientation would provide a stronger reference for computing orientation contrast. Of course, "grouping" remains a possibility, though in this version it would be controlled top-down for a color-based set.

Interestingly, whereas the effect of distractor repetition was independent of the number of display elements on target-absent trials (though there was a tendency for an interaction), on target-present trials, the distractor repetition effect did depend on display size: It was greater (and only reliable) with 8- and 16-element displays. At first glance, this display size $\times$ target interaction might be taken to suggest that priming results from different mechanisms on target-present trials (e.g., priming resulting from repetition of target orientation) and target-absent trials (e.g., priming resulting from repetition of distractor orientation). However, the nonreliable facilitation in 4-element displays for the target-present trials could equally be explained by assuming that the grouping of distractors, and thus faster target discernment, was less efficient with 4 than with 8 or 16 display elements. In particular, with only 4 elements in the display, there were only two, differently oriented red items: one target and one distractor.

Consider, for example, a pair of consecutive trials in which the display contains, on the first trial, a horizontal red target, one vertical red distractor, and two horizontal green distractors; on the next, the display contains a vertical red target, one horizontal red distractor, and two vertical green distractors. Now assume that, on target-present trials, the facilitatory effect of distractor repetition results from prioritized grouping of red distractors (see note 1). When two differently oriented red elements are in the display (one of them the target), there are two ways of making a "target-present" decision, by either detecting an orientation difference between the red elements or checking which of the two red elements matches the orientation of the green distractors. Given that search operates via the (enhanced grouping of ) red elements, the former possibility may be more likely. As a result of this, the red distractor might be erroneously selected as the target on some trials (on others, the target is correctly selected). On the next trial, on which both the (red) target and the (red) distractor orientations are changed (dTdD), there may be a bias toward selecting as the target (in this case, correctly) the red element that shares the same orientation as the mistaken target on the preceding trial (Figure 4). This bias - in effect, a form of target-on-target priming - would reduce the RT disadvantage usually found in the $\mathrm{dTdD}$ condition; in other words, it would reduce the amount of facilitation usually observed in the sTsD and dTsD conditions (relative to the dTdD condition). In contrast, the 8- and 16element displays contained, respectively, three and seven red distractors, so the uniquely oriented target was unlikely to be misselected as a distractor, and large RT advantages were produced for $\mathrm{sTsD}$ and $\mathrm{dTsD}$ conditions relative to the sTdD and dTdD conditions. (In some sense, this account resembles that put forward by Bravo \& Nakayama, 1992, for the prolonged "pop-out" search RTs they observed with 2-element displays; in such displays, it was not immediately clear which of the two elements was the target.)

Some support for the account above (of less efficient target selection between two red items in 4-element displays) may also be found in the Kristjánsson et al. (2002) study, in which the effects of repeated target and distractor orientations were somewhat smaller for 4-element $(8 \mathrm{msec})$ than for 8 - and 16 -element displays $(\approx 12 \mathrm{msec})$. Nevertheless, we should mention two points of difference: First, the design of the Kristjánsson et al. study did not permit them to distinguish the effects of repeated target and distractor orientations; thus, the larger priming 
effects with 8- and 16-element displays may have been confounded by repetition of the target orientation, rather than due to repetition of the distractor orientation alone. Second, in the present experiments, the priming effects found in the sTdD, dTsD, and sTsD conditions, which averaged 16,35 , and $35 \mathrm{msec}$, respectively, were much larger than those reported by Kristjánsson et al. One possible explanation for the stronger priming effects could be that there were four (present experiment) rather than just two (Kristjánsson et al., 2002) different target or distractor orientation alternatives. This would have increased the uncertainty associated with the possible orientation of the target or the distractors on a given trial, which in turn could have increased the RT advantage when the red display elements (target, distractors, or both) were in the same orientation on the next trial.

Support for this suggestion is provided by our analysis of the present data based on the number of possible target versus distractor alternatives. Although the target- and distractor-based facilitation effects revealed in Experiments $1 \mathrm{~A}$ and $1 \mathrm{~B}$ were relatively unaffected by the number of target alternatives, the number of (red) distractor alternatives had a discernible effect: The overall facilitation relative to preceding trials $n-1$ and $n-2$ was larger, by $10 \mathrm{msec}(n-1)$ and $44 \mathrm{msec}(n-2)$, when the number of distractor alternatives was four rather than just two (Experiment $1 \mathrm{~A}$ vs. 1B). Thus, the overall dominant distractor priming effect is affected by distractor uncertainty, perhaps because the discernment of target presence requires a more detailed (i.e., time-consuming) distractor analysis when the number of distractor alternatives is increased; this, in turn, would strengthen the "set" for the current type of distractor, leading to stronger priming when this distractor type is repeated (see Müller et al., 2004, for a similar explanation of an increased target-based priming effect under singleton feature search conditions that required detailed analysis of target identity, rather than simply target detection). However, this suggestion is tentative, requiring further investigation.

\section{GENERAL DISCUSSION}

Several mechanisms have been proposed that could account for efficient visual search for conjunctively defined targets: inhibition of distractor features (Treisman \& Sato, 1990), enhancement of target features (Wolfe et al., 1989), feature dissimilarity between target and distractors contrasted with similarity among distractors (Duncan \& Humphreys, 1989), or, in the context of even relatively efficient conjunction searches, the priming of target and/or distractor features across consecutive trials (Kristjánsson et al., 2002; Wang et al., 2001, 2005). The present results provide further evidence in favor of the latter account; in addition, they demonstrate that priming derives mainly from the repetition of distractor, rather than target, features. (Note, however, that Weidner et al., 2002, found large target-based priming effects in a conjunction search task in which the target was defined by a conjunction of a constant primary dimension, size, and an across-trials variable secondary dimension, color or motion. In this case, the facilitation deriving from repetition of the secondary target dimension was in excess of $100 \mathrm{msec}$, which compared with a facilitation effect of approximately $16 \mathrm{msec}$ when the target-defining feature within the secondary dimension was repeated.)

\section{A Memory System for Priming in Visual Search}

Given that the repetition of display elements' features across trials can improve search efficiency, how can the memory underlying the feature priming be characterized? One possibility is that it works via an automatic, topdown impenetrable, implicit visual short-term memory (Maljkovic \& Nakayama, 1994, Experiment 4). However, recent work by Müller and his colleagues (Müller et al., 2004; Müller et al., 2003) suggests that this may not be the whole truth. For example, in a singleton feature search task, Müller et al. (2004) found evidence that automatic priming can be influenced by factors associated with the task set. When participants had to explicitly encode (and retain) the target-defining dimension or feature, dimensionspecific cross-trial facilitation effects (see Müller, Heller, \& Ziegler, 1995) increased relative to a no-control condition in which encoding was not required; note, though, that priming (of reduced magnitude) was also manifest in the latter condition. The increased cross-trial facilitation in the "encode" conditions was taken as evidence for top-down modification of a (dimension-specific) visual short-term memory system that, in default mode, operates in a largely automatic fashion.

Perhaps these discrepant results suggest independent priming mechanisms, one operating at the feature level (see, e.g., Maljkovic \& Nakayama, 1994), which is topdown impenetrable, and one at the dimensional level (see, e.g., Müller et al., 2004; Müller et al., 2003), which is sensitive to top-down biasing.

Another explanation is suggested by Hillstrom (2000). In her Experiment 1, participants responded to the orientation of a color singleton target that, in one condition, changed its color predictably every two trials. The results were similar to those reported by Maljkovic and Nakayama (1994, Experiment 4), in that an RT advantage was found when the color of the target was repeated on consecutive trials. However, the search RTs were overall faster, by $115 \mathrm{msec}$, when the target color changed predictably rather than randomly across trials, a finding interpreted by Hillstrom (2000, p. 803) as an effect of top-down "expectancy"- - at variance with Maljkovic and Nakayama, 1994. Priming effects were also evident when an additional color singleton (a distractor) was presented: It prevented observers from using a simple saliency-based search strategy and instead required them to set themselves, on a trialby-trial basis, for a particular feature (Experiment 3); or, alternatively, when they had to set themselves for a particular conjunction of target features (Experiment 4), it required them to adopt a template-based search strategy (see Duncan \& Humphreys, 1989). Hillstrom took the finding of priming effects in a wide variety of search tasks (from singleton feature search to cued feature and conjunction 
search) to suggest that a single memory mechanism may be responsible for these effects. Interestingly, in the conjunction search task, repetition did not affect the search time per element (the slope of the search RT/display size function), but rather the base RT ( $y$-intercept of the function). This was also the case in the present experiments, in which the search times were similar in the sTsD, sTdD, dTsD, and $\mathrm{dTdD}$ conditions $(15.4,15.1,16.5$, and $18.4 \mathrm{msec} /$ element, respectively) to those of Weidner et al. (2002). Hillstrom (p. 811) took this to suggest that repetition affects the speed of the spatially parallel enhancement (in Hillstrom's terms, "prioritizing") of target feature coding (see Wolfe, 1994).

On the basis of these findings, Hillstrom (2000) proposed an episodic memory mechanism of "prioritization": If the target features determining selection on a given trial are repeated, a memory trace of the priorities assigned to the display elements on this trial can be carried over to the next trial, expediting the processing of items sharing target features in the new display. In contrast, if the target features change, a new set of priorities must be established, leading to RT costs. Note that this episodic-memory explanation in some sense resembles the "weighting" account proposed by Müller and colleagues (see, e.g., Müller et al., 1995; Müller et al., 2003). fMRI data suggest that one component of the "episodic" memory is realized in terms of sustained enhancement of feature-coding mechanisms in extrastriate visual areas (see, e.g., Pollmann, Weidner, Müller, \& von Cramon, in press).

However, with regard to Hillstrom's (2000) account, the present findings suggest that it is not only, or even foremost, episodic memory for target features that determines the speed of prioritizing the processing of the new display elements; rather, it is episodic memory for distractor features that carries a greater weight.

\section{REFERENCES}

Bacon, W. F., \& Egeth, H. E. (1997). Goal-directed guidance of attention: Evidence from conjunctive visual search. Journal of Experimental Psychology: Human Perception \& Performance, 23, 948-961.

Bravo, M. J., \& NAKAYAMA, K. (1992). The role of attention in different visual-search tasks. Perception \& Psychophysics, 51, 465-472.

Driver, J., McLeod, P., \& Dienes, Z. (1992). Motion coherence and conjunction search: Implications for guided search theory. Perception \& Psychophysics, 51, 79-85.

DunCAN, J., \& Humphreys, G. W. (1989). Visual search and stimulus similarity. Psychological Review, 96, 433-458.

Duncan, J., \& HumphreYs, G. W. (1992). Beyond the search surface: Visual search and attentional engagement. Journal of Experimental Psychology: Human Perception \& Performance, 18, 578-588.

Egeth, H. E., Virzi, R. A., \& Garbart, H. (1984). Searching for conjunctively defined targets. Journal of Experimental Psychology: Human Perception \& Performance, 10, 32-39.

Enns, J. T., \& Rensink, R. A. (1991). Preattentive recovery of threedimensional orientation from line drawings. Psychological Review, 98, 335-351.

Friedman-Hill, S. R., \& Wolfe, J. M. (1995). Second-order parallel processing: Visual search for the odd item in a subset. Journal of Experimental Psychology: Human Perception \& Performance, 21, 531-551.

He, Z. J., \& Nakayama, K. (1992). Surfaces versus features in visual search. Nature, 359, 231-233.
Hillstrom, A. P. (2000). Repetition effects in visual search. Perception \& Psychophysics, 62, 800-817.

Humphreys, G. W., \& Müller, H. J. (1993). SEarch via Recursive Rjection (SERR): A connectionist model of visual search. Cognitive Psychology, 25, 43-110.

Kaptein, N. A., Theeuwes, J., \& van der Heijden, A. H. C. (1995). Search for a conjunctively defined target can be selectively limited to a color-defined subset of elements. Journal of Experimental Psychology: Human Perception \& Performance, 21, 1053-1069.

KARNI, A., \& SAGI, D. (1993). The time course of learning a visual skill. Nature, 365, 250-252.

Kingstone, A., \& Bischof, W. F. (1999). Perceptual grouping and motion coherence in visual search. Psychological Science, 10, 151-156.

KristuánsSon, Á., WANG, D., \& NAKAYAMA, K. (2002). The role of priming in conjunctive visual search. Cognition, 85, 37-52.

Maljkovic, V., \& NakaYama, K. (1994). Priming of pop-out: I. Role of features. Memory \& Cognition, 22, 657-672.

Maljkovic, V., \& NaKayama, K. (1996). Priming of pop-out: II. The role of position. Perception \& Psychophysics, 58, 977-991.

Maljkovic, V., \& Nakayama, K. (2000). Priming of pop-out: III. A short-term implicit memory system beneficial for rapid target selection. Visual Cognition, 7, 571-595.

McPeek, R. M., Maljkovic, V., \& Nakayama, K. (1999). Saccades require focal attention and are facilitated by a short-term memory system. Vision Research, 39, 1555-1566.

Müller, H. J., Heller, D., \& Ziegler, J. (1995). Visual search for singleton feature targets within and across feature dimensions. Perception \& Psychophysics, 57, 1-17.

Müller, H. J., Krummenacher, J., \& Heller, D. (2004). Dimensionspecific intertrial facilitation in visual search for pop-out targets: Evidence for a top-down modulable visual short-term memory effect. Visual Cognition, 11, 577-602.

Müller, H. J., Reimann, B., \& Krummenacher, J. (2003). Visual search for singleton feature targets across dimensions: Stimulusand expectancy-driven effects in dimensional weighting. Journal of Experimental Psychology: Human Perception \& Performance, 29, 1021-1035.

Nakayama, K., \& Silverman, G. H. (1986). Serial and parallel processing of visual feature conjunctions. Nature, 320, 264-265.

Pollmann, S., Weidner, R., Müller, H. J., \& von Cramon, D. Y. (in press). Neural correlates of visual dimension weighting. Visual Cognition.

Ramachandran, V. S. (1988). Perception of shape from shading. Nature, 331, 163-166.

Segalowitz, S. J., \& Graves, R. E. (1990). Suitability of IBM XT, $\mathrm{AT}$, and PS/2 keyboard, mouse, and game port as response devices in reaction time paradigms. Behavior Research Methods, Instruments, \& Computers, 22, 283-289.

Treisman, A., \& Gelade, G. (1980). A feature-integration theory of attention. Cognitive Psychology, 12, 97-136.

Treisman, A., \& Sato, S. (1990). Conjunction search revisited. Journal of Experimental Psychology: Human Perception \& Performance, 16, 459-478.

VON MüHLENEN, A., \& MüLleR, H. J. (2001). Visual search for motionform conjunctions: Is form discriminated within the motion system? Journal of Experimental Psychology: Human Perception \& Performance, 27, 707-718.

WANG, D., KRISTJÁNSSON, Á., \& NAKAYAMa, K. (2001). Efficient visual search without top-down or bottom-up guidance: A putative role for perceptual organization (Tech. Rep. No. 26). Columbus: Ohio State University, Center for Cognitive Science.

Wang, D., Kristjánsson, Á., \& Nakayama , K. (2005). Efficient visual search without top-down or bottom-up guidance. Perception \& Psychophysics, 67, 239-253.

Weidner, R., Pollmann, S., Müller, H. J., \& von Cramon, D. Y. (2002). Top-down controlled visual dimension weighting: An eventrelated fMRI study. Cerebral Cortex, 12, 318-328.

Wolfe, J. M. (1994). Guided search 2.0: A revised model of visual search. Psychonomic Bulletin \& Review, 1, 202-238.

Wolfe, J. M. (1998). Visual search. In H. Pashler (Ed.), Attention (pp. 13-73). Hove, U.K.: Psychology Press. 
Wolfe, J. M., Cave, K. R., \& Franzel, S. L. (1989). Guided search: An alternative to the feature integration model for visual search. Journal of Experimental Psychology: Human Perception \& Performance, $15,419-433$

\section{NOTE}

1. Evidence for the proposal that the facilitatory effects in Experiments $1 \mathrm{~A}$ and $1 \mathrm{~B}$ resulted from the repetition of the red distractors (i.e., essentially, subset search of the red elements) was provided by a control experiment. In this experiment, observers had to search for a uniquely oriented red target. However, in contrast to Experiments 1A and 1B, the orientation of the red distractors was orthogonally coupled with that of the red target (e.g., when the target orientation was horizontal, the orientation of the red distractors was vertical, and vice versa), and the orientation of the green distractors varied independently of the target orientation. It was hypothesized that, if performance was dependent on subset search of the red display elements, search RTs would be influenced only by repetition/ change of the red distractors' orientation, not by variation of the green distractors' orientation.

The target-present RT data were analyzed by means of an ANOVA with a single factor-namely, cross-trial transition - with reference to both the red target and the green distractor orientation: same target orientation, same green distractor orientation (sTsD); same target orientation, different green distractor orientation (sTdD); different target orientation, same green distractor orientation (dTsD); and different target orientation, different green distractor orientation (dTdD). (Since the orientation of the red distractors was tied with that of the target, the red distractors' orientation was repeated/changed whenever the orientation of the target was repeated/changed.) This ANOVA revealed a significant effect of cross-trial transition $\left[F(3,27)=3.68, M S_{\mathrm{e}}=1,383, p<.05\right]$. RTs were faster in the sTsD and sTdD conditions ( 710 and $719 \mathrm{msec}$, respectively) than in the dTsD and dTdD conditions ( 750 and $756 \mathrm{msec}$, respectively). This pattern means that only repetition of the red target- that is, of the red distractors, orthogonally oriented relative to the target-produced a significant facilitatory effect (of approximately $40 \mathrm{msec}$; Tukey LSD tests revealed RTs both in the sTsD and sTdD conditions to be faster than in the $\mathrm{dTdD}$ condition); repetition of the freely varying green distractors did not (facilitation $<10 \mathrm{msec}$, with no difference in RTs between the dTsD and dTdD conditions on a Tukey LSD test).

(Manuscript received November 10, 2004; revision accepted for publication July 28, 2005.) 